\title{
The Diagnostic Function of Optical Coherence Tomography in Diabetic Maculopathy
}

\author{
Authors: Gehad Mahmoud, Prof.Dr.Gamal abdelatif, Assist.Prof.Dr.Mohamed \\ Alagouz and Dr.Mahmoud Farouk. \\ Ophthalmology Department, Faculty of Medicine Sohag University
}

Diabetic maculopathy (DM) is one of the major causes of vision impairment in individuals with diabetes. The traditional approach to diagnosis of DM includes fundus ophthalmoscopy and fluorescein angiography. Although very useful clinically, these methods do not contribute much to the evaluation of retinal morphology and its thickness profile. That is why a new technique called optical coherence tomography (OCT) was utilized to perform cross-sectional imaging of the retina. It facilitates measuring the macular thickening, quantification of diabetic macular edema, and detecting vitreoretinal traction. Thus, OCT may assist in patient selection with DM who can benefit from treatment, identify what treatment is indicated, guide its implementing, and allow precise monitoring of treatment response. It seems to be the technique of choice for the early detection of macular edema and for the follow up of DM.

\section{Introduction ..}

Optical coherence tomography (OCT) enables precise measurement of macular thickness. Thus, it facilitates detecting macular edema which is the main pathologic feature of diabetic maculopathy. This is defined as any detectable retinal thickening due to fluid accumulation. The edema may be symmetrical or involve only a sector of the macular area. It usually starts as a focal lesion and progresses towards a more diffuse form. In some cases, the macular edges may be thickened, even though the contour of the foveal centre remains normal. Persistent retinal edema resulting in Muller cell necrosis leads to the formation of cystoid cavities, located mainly in the outer retina and sometimes also in the inner plexiform layer. In the most advanced stages in eyes with well established long term macular edema, several central cysts can merge together forming large hyporeflective cavity which contributes to the significant thickening of the fovea (Figure 1). Therefore, the main characteristics of macular edema in OCT, apart from increased retinal thickness, include intraretinal spaces of reduced reflectivity, disintegration of the layered retinal structure, and usually also flattening of the central foveal depression. In some cases fluid can be seen under the neurosensory retina [1]. OCT tomograms can also reveal hard exudates and haemorrhages. They present as small hyperreflective deposits with posterior shadowing intraretinal cysts can differ in size. That is why Koleva- Gorgieva proposed the classification of cystoid diabetic macular edema (DME) into mild, moderate, and severe according to the size of cystoid spaces [2]. 


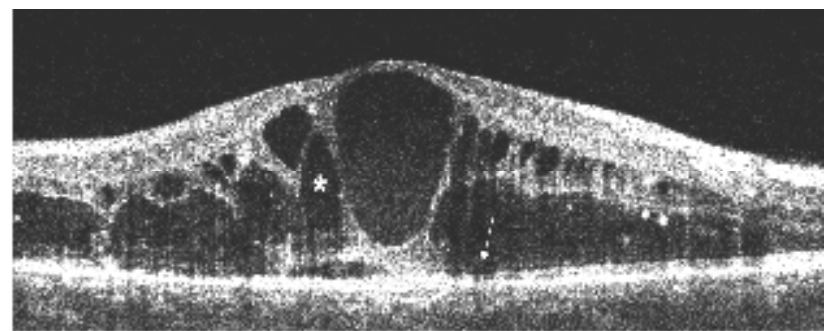

Figure 1: Cystoid DME: SDOCT demonstrating hyporeflective cystic spaces within the retina (white asterisk) and subretinal fluid accumulation consistent with macular edema.

The mild cystoids DME presents with small cysts predominantly in the outer retinal layers. The cystoid spaces in eyes with intermediate and severe cystoid DME are mainly located in the outer layers, predominantly in the fovea. In some cases small cysts in the inner layers can also be found. If the cysts continue to increase, they may occupy the full thickness of the retina, leading to its atrophy and the profound vision loss. In the past few years, since the introduction of FDOCT, it has become possible to accurately visualize the outer retinal layers. The integrity of these layers has been shown to correlate with retinal function. Several authors have reported that the integrity of ELM and IS/OS junction has a positive correlation with visual acuity [3,4]. Shin et al. showed that the photoreceptor layer status is closely associated with final visual acuity in DME and that photoreceptor integrity prior to treatment can be predictive of potential visual recovery in DME [5]. Yohannan et al. demonstrated that disruption of IS/OS junction correlates well with a significant decrease in point sensitivity in eyes with DME [6].Therefore, the assessment of outer retinal layer structure should be a part of a routine evaluation when performing OCT in patients with DME. The ability to visualize the vitreoretinal interface is a unique feature of OCT. It allows for macular traction imaging, which may play a role in DME development [7, 8]. The traction may be induced by vitreoretinal interface abnormalities such as incomplete posterior vitreous detachment (PVD) or epiretinal membrane (ERM). If the posterior hyaloid is thin and only slightly detached from the surface of the macula, it is not visible in ophthalmoscopy, but can be easily detected by OCT. The same is true for ERM; if it is thin and does not cause a significant retinal distortion, it can be only visualized using OCT. The detection of clinically significant macular traction may affect therapeutic management of DME. Releasing the traction during vitrectomy may be the best treatment option in those patients [9].That is why the assessment of vitreoretinal interface is an essential step in macular evaluation in patients with diabetic retinopathy. Moreover, OCT does not only work well as a diagnostic tool in macular traction but may also be used in order to monitor the postoperative morphological outcomes. It can also help identify the postoperative complications of vitrectomy, such as retinal detachment, ERM, and lamellar macular hole formation. The detached posterior vitreous face presents OCT scans as a thin hyper-to-medium reflective horizontal or oblique line in the nonreflective vitreous cavity, above or inserting into the retina. In case of incomplete PVD it may adhere to the foveal or the peripapillary region [10]. ERM on OCT scans presents as a hyperreflective line lying on retinal surface. It can lead to increase in 
macular thickness, loss of foveal depression, and formation of intraretinal cystoids spaces or pseudo holes. The distinction between ERMs and a PVD is usually made on the basis of reflectivity. The latter typically has a lower reflectivity and less consistent appearance than preretinal fibrosis. OCT can also be used to document the opacity and thickness of ERM, its distance from the surface of the retina, and such effect on the underlying retina as distortion, edema, or neurosensory detachment. It should be noted that OCT is complementary to ultrasound scanning in evaluation of vitreoretinal interface. Ultrasound scans provide a more complete image of vitreous pathology but at the cost of lower resolution. FDOCT, on the other hand, provides a more detailed image of vitreoretinal interface but limited to a relatively small area. As OCT uses light to acquire the images, if the optic media are opacified and fundus cannot be visualized, the retinal cross sections will not be obtained. The limitations are similar to the ones associated with ophthalmoscopy and FA. However, Patients \& Methods..

This study was carried out between November 2015 to March 2017 and included 500 eyes of

287 patients with different stages of diabetic retinopathy ,115 males and 172 females. Their ages ranged from 20 to 80 years. They were attending the retina clinic in Sohag University Hospital. They were candidate for Optical Coherence Tomography (OCT).

\section{- Study design:}

A prospective, non-comparative, noninterventional, case series study.

\section{- Inclusion criteria:}

All patients with diabetic maculopathy attended the retina clinic in Sohag University sometimes OCT images can be acquired in cases where retinal assessment in ophthalmoscopy is impossible. In some cases OCT can even enable assessment of the space located posteriorly to the thin fibrovascular membrane in proliferative retinopathy. Another particular value of OCT is the possibility of reliable and reproducible retinal thickness measurements. Using the retinal thickness maps, it is possible to monitor DME progression and assess treatment outcomes after laser photocoagulation, intravitreal injections of anti- VEGF and steroids or, as mentioned before, vitrectomy. The obtained results can be compared with the normative database. Owing to retinal thickness maps not only edema but also atrophy can be detected, which contributes to lack of improvement or even decreased vision after the edema is resolved. The treatment efficacy in DME should, then, be evaluated in terms of two outcomes: the functional one based on visual acuity measurements and anatomical one assessed in OCT.[8]

Hospital were grouped and analyzed according to the OCT findings.

\section{- Exclusion criteria:}

Patients who were suffering from other retinal vascular diseases as retinal vein occlusion,

retinal artery occlusion and retinal vasculitis were excluded from this study.

\section{- Methods:}

All patients were subjected to: A) History taking including : Name, Age, Gender, Duration of diabetes mellitus ,Type of medication for diabetes, Associated systemic diseases (hypertension, renal and cardiac diseases), Past history of ocular surgery or LASER therapy and Ocular medication. 
B) Examination: including: Visual acuity (un-corrected \& best corrected), Anterior segment examination by slit lamp biomicroscopy, IOP measurement, Fundus examination by direct, indirect ophthalmoscopy and slit lamp biomicroscopy.

C) Investigations: Optical coherence tomography (OCT) and fluorescein angiography.

- Main outcomes measures: The findings obtained from OCT.

Eyes were assessed and classified according to OCT findings of retinal thickness, retinal

morphology, retinal topography, macular traction, and foveal photoreceptor status . This

classification was described by Koleva-Georgieva.[11]. This includes the following:

\section{Results..}

The present study included 500 eyes of diabetic maculopathy patients in different stages of

diabetic retinopathy ( 115 males \& 172 females ). There ages ranged from $20-80$ years. They were candidates for OCT. The duration of the diagnosed diabetes range from $3-19$ years. They were on different hypoglycemic medications, 169 patients were on insulin while 116 patients were on oral hypoglycemic drugs and 2 patients were on combination of insulin and hypoglycemic drugs. Some patients were suffering from systemic disorders that may affect the condition of diabetic retinopathy, as systemic hypertension in 227 patients, ischemic heart diseases in 72 patients, renal disorders in 27 patients and smoking in 58 patients. Diabetic maulopathy was diagnosed in $100 \%$ of included eyes, with macular edema in 286 patients $(57.2 \%)$, ischemic maculoathy in 94 patients (18.8\%) and mixed maculopathy in 120 patients ( $24 \%$ ).

\section{Conclusion..}

OCT can perform micrometreresolution, cross-sectional imaging of the retina that closely approximates its histological layers. One of the huge advantages of OCT is that patients find this procedure very comfortable because it is non contact and the measurement time is very short. In patients with diabetic retinopathy OCT can be successfully utilized as an objective monitoring technique of the macular thickening before and after therapy. Thus, it facilitates quantification of retinal edema. OCT is also very useful for vitreous assessment, showing whether it is attached or detached from the macula. It is helpful in detecting vitreoretinal traction that may not have been identified clinically. To summarize, OCT may assist in patient selection with diabetic maculopathy who can benefit from treatment, identify what treatment is indicated, guide its implementing, and allow precise monitoring of treatment response. OCT also helps to understand the anatomy of DME and the intraretinal damage. It 
seems to be the technique of choice for the early detection of macular edema and for the follow up of diabetic maculopathy.

\section{References..}

1-D. Koleva-Georgieva and N. Sivkova, "Assessment of serous macular detachment in eyes with diabetic macular edema by use of spectraldomain optical coherence tomography," Graefe's Archive for Clinical and Experimental Ophthalmology, vol. 247, no. 11, pp. 1461-1469, 2009.

2-D. N. Koleva-Georgieva and N. P. Sivkova, "Types of diabetic macular edema assessed by optical coherence tomography," Folia Medica, vol. 50, no. 3, pp. 30-38, 2008.

3-T. Otani, Y. Yamaguchi, and S. Kishi, "Correlation between visual acuity and foveal microstructural changes in diabetic macular edema," Retina, vol. 30, no. 5, pp. 774-780, 2010.

4-A. S. Maheshwary, S. F. Oster, R. M. S. Yuson, L. Cheng, F. Mojana, andW. R. Freeman, "The association between percent disruption of the photoreceptor inner segment-outer segment junction and visual acuity in diabetic macular edema, "American Journal of Ophthalmology,vol.150, no.1, pp.63.e167.e1,2010.

5-H. J. Shin, S. H. Lee, H. Chung, and H. C. Kim, "Association between photoreceptor integrity and visualoutcome in diabetic macular edema," Graefe's Archive for Clinical and Experimental Ophthalmology, vol. 250, no. 1, pp. 61-70, 2012.
6-J. Yohannan, M. Bittencourt, Y. J. Sepah et al., "Association of retinal sensitivity to integrity of photoreceptor inner/outer segment junction in patients with diabetic macular edema," Ophthalmology, vol. 120, pp. 12541261, 2013.

7- J. I. Patel, P. G. Hykin, M. Schadt, V. Luong, F. Fitzke, and Z. J. Gregor, "Pars plana vitrectomy for diabetic macular oedema: OCT and functional correlations," Eye, vol. 20, no. 6, pp. 674-680, 2006.

8-S.D. Pendergast, T. S.Hassan, G. A.Williams et al., "Vitrectomy for diffuse diabetic macular edema associated with a taut premacular posterior hyaloid," American Journal of Ophthalmology, vol. 130, no. 2, pp. 178-186, 2000.

9- S. P. Shah, M. Patel, D. Thomas, S. Aldington, and D. A. H. Laidlaw, "Factors predicting outcome of vitrectomy for diabetic macular oedema: results of a prospective study," British Journal of Ophthalmology, vol. 90, no. 1, pp. 3336, 2006.

10- D. Gaucher, R. Tadayoni, A. Erginay, B. Haouchine, A. Gaudric, and P. Massin, "Optical coherence tomography assessment of the vitreoretinal relationship in diabetic macular edema," American Journal of Ophthalmology, vol. 139, no. 5, pp. 807-813, 2005.

11-D. Koleva-Georgieva, "Optical coherence tomography findings in diabetic macular edema," in Diabetic Retinopathy, M. S. Ola, Ed., InTech, Vienna, Austria, 2012. 
SOHAG MEDICAL JOURNAL The Diagnostic Function of Optical Coherence Tomography 